\title{
Alisertib induces apoptosis and autophagy through targeting the AKT/mTOR/AMPK/p38 pathway in leukemic cells
}

\author{
YUNFENG FU $^{1 *}$, YANAN ZHANG ${ }^{1 *}$, MENG GAO $^{1}$, LINGLI QUAN ${ }^{2}$, RONG GUI ${ }^{1}$ and JING LIU ${ }^{1}$ \\ ${ }^{1}$ The Third Xiangya Hospital, Central South University, Changsha, Hunan 410013; \\ ${ }^{2}$ The First Department of Respiratory Medicine, Central Hospital of Zhuzhou, Zhuzhou, Hunan 412000, P.R. China
}

Received March 25, 2015; Accepted February 16, 2016

DOI: $10.3892 / \mathrm{mmr} .2016 .5249$

\begin{abstract}
Alisertib, a potent and selective Aurora kinase A inhibitor, has been demonstrated to exert potent anti-cancer effects in pre-clinical and clinical studies. However, mechanisms of action of alisertib, including the molecular pathways involved in alisertib-induced apoptosis and autophagy of leukemic cells, have remained elusive. The aim of the present study was to investigate the effects of alisertib on cell growth, apoptosis and autophagy and to delineate the possible molecular mechanisms in leukemic cells. Acid phosphatase, MTT and Annexin V/propidium iodide staining assays as well as immunostaining for light chain $3 \mathrm{~B}$ showed that treatment of the REH leukemia cell line with alisertib exerted potent growth inhibitory effects, and induced apoptosis and autophagy in a dose-dependent manner. Western blot analysis indicated that these effects may be attributed to the suppression of the activity of the Akt/mammalian target of rapamycin/5'-AMP-dependent kinase/p38 mitogen-activated protein kinase signaling pathways in REH cells. The present study confirmed that alisertib may represent a promising autophagy-inducing drug for the treatment of leukemia and shed light on its molecular mechanism of action.
\end{abstract}

\section{Introduction}

Leukemia is a hematopoietic malignancy caused by acquired somatic mutation and ranks among the ten types of malignant neoplasm with the highest incidence rate. Chronic myeloid leukemia is a common hematopoietic malignancy, accounting for $20 \%$ of all types of leukemia (1). Cancer fusion protein

Correspondence to: Mr. Rong Gui or Mr. Jing Liu, The Third Xiangya Hospital, Central South University, 138 Tongzipo Road, Changsha, Hunan 410013, P.R. China

E-mail: rongggrong@163.com

E-mail: jjliuliuyswq@126.com

*Contributed equally

Key words: alisertib, autophagy, leukemic cells, Akt, mammalian target of rapamycin p210bcr/abl generated by $\mathrm{Ph}$ chromosome has an important role in these malignant phenotypes, as it has been demonstrated to induce leukemia-like syndrome in cancerous mice, to promote tumor-cell proliferation and survival in vitro as well as to be implicated in the evasion of apoptosis of tumor cells (2).

Autophagy is a conserved self-degradation system in eukaryotic cells involved in numerous physiological and pathological processes. Autophagosomes are a typical feature of autophagy, and the control of their formation and degradation is the main regulatory factor in autophagy (3). Autophagy has a dual nature of promoting cell survival and death, and is also closely associated with the development, metastasis and drug resistance of cancer (4). Targeting autophagy may be a novel strategy to treat cancer and address drug resistance. In-depth study of autophagy in leukemia will lead to the elucidation of the induction and regulatory mechanisms of leukemia-cell autophagy and provide novel targets and strategies for leukemia treatment and possible cures (5).

The mechanisms of action of the promising anti-cancer drug candidate alisertib are complex and have remained to be fully elucidated. As alisertib has been demonstrated to induce apoptosis (6), the present study assessed the underlying molecular mechanisms. A recent study showed that alisertib exerts pro-autophagic effects on human osteosarcoma cell lines through the activation of a mitochondria-mediated pathway and inhibition of the p38 mitogen-activated protein kinase (MAPK)/phosphoinositide-3 kinase (PI3K)/Akt/mammalian target of rapamycin (mTOR) signaling pathway (7). To assess whether alisertib exerts its effects against leukemia cells via similar mechanisms, its ability to inhibit proliferation, induce autophagy and affect MAPK/PI3K/Akt/mTOR signaling were assessed in the REH leukemia cell line.

\section{Materials and methods}

Reagents. Dulbecco's modified Eagle's medium (DMEM) and fetal bovine serum (FBS) were obtained from Mediatech Inc. (Manassas, VA, USA). Alisertib (chemical structure shown in Fig. 1) was purchased from Selleckchem Inc. (Houston, TX, USA). Acid phosphatase assay (AP) and 3-(4,5-dimethylthiazol-2-yl)-2,5-diphenyltetrazolium bromide (MTT) were from Invitrogen (Thermo Fisher Scientific, Inc., Waltham, MA, USA). Annexin V-fluorescein isothiocyanate (FITC) and propidium iodide (PI) were obtained from BD Biosciences (San Diego, CA, 
USA). All antibodies used for western blot analysis were from Santa Cruz Biotechnology, Inc. (Dallas, TX, USA).

Cell lines and culture. The REH human acute lymphocytic leukemia cell line was purchased from Central South University (Hunan, China). REH cells were cultured in DMEM medium supplemented with $2 \mathrm{mM}$ L-glutamine and $1 \%$ of antibiotic/antimycotic mixture (Sigma-Aldrich, St. Louis, MO, USA). Cells were maintained in a humidified atmosphere of $5 \% \mathrm{CO}_{2} / 95 \%$ air at $37^{\circ} \mathrm{C}$.

Cell viability assay. Cells were seeded into 96-well plates at a density $1.0-2.0 \times 10^{4}$ cells/well and treated with alisertib $(0,0.1,1$ or $5 \mu \mathrm{M})$ for $24 \mathrm{~h}$. For the PA assay, the cells were subsequently incubated for $2 \mathrm{~h}$ at $37^{\circ} \mathrm{C}$ with $100 \mathrm{ml}$ p-nitrophenyl phosphate solution $(5 \mathrm{mM})$ and $0.1 \%$ Triton $\mathrm{X}-100$. The reaction was terminated by addition of $10 \mathrm{ml} \mathrm{NaOH}(1 \mathrm{M})$ and the number of viable cells was measured at $405 \mathrm{~nm}$ using a Multiskan Spectrum microplate reader (Thermo Fisher Scientific, Inc.). For the MTT assay, $20 \mu \mathrm{l}$ MTT was added to the cells, which were then cultured for an additional $4 \mathrm{~h}$. Following aspiration of the MTT solution, dimethylsulfoxide (Nanjing Chemical Reagent Co., Ltd., Nanjing, China) was added to each well and plates were agitated for $20 \mathrm{~min}$. The absorbance was then measured with Multiskan Spectrum microplate reader at $490 \mathrm{~nm}$.

Apoptosis assay. Cells were seeded into six-well plates at a density $1.0-2.0 \times 10^{6}$ cells/well and treated with alisertib $(0$, $0.1,1$ and $5 \mu \mathrm{M}$ ) for $24 \mathrm{~h}$. Following harvesting, cells were washed twice with ice-cold phosphate-buffered saline (PBS), re-suspended in binding buffer and then incubated with $10 \mu \mathrm{l}$ Annexin V-FITC and $5 \mu \mathrm{l}$ PI in the dark for $30 \mathrm{~min}$. The samples were then analyzed by flow cytometry (FACSAria III; BD Biosciences).

Immunofluorescence microscopy. Cells were seeded into six-well plates at a density $1.0-2.0 \times 10^{6}$ cells/well and treated with alisertib $(0,0.1,1$ and $5 \mu \mathrm{M})$ for $24 \mathrm{~h}$. Cells were then fixed with $4 \%$ paraformaldehyde (Solarbio Science and Technology, Ltd., Beijing, China) for $30 \mathrm{~min}$ at room temperature and permeabilized using pre-cooled methanol on ice for $10 \mathrm{~min}$. Binding sites were then blocked with 5\% normal goat serum (Thermo Fisher Scientific, Inc.) and 0.3\% Triton X-100 (Thermo Fisher Scientific, Inc.) for $1 \mathrm{~h}$. Cells were then incubated with mouse monoclonal anti-light chain (LC)3B (cat. no. sc-376404; 1:300; Santa Cruz Biotechnology, Inc.) followed by $1 \mathrm{~h}$ of incubation with Alexa Fluor 488 goat anti-mouse immunoglobulin G (cat. no. 8878; 1:1,000 dilution; Cell Signaling Technology, Inc., Danvers, MA, USA) as a secondary antibody. After incubation, cells were washed with ice-cold PBS, mounted onto microscopic slides with Flouromount-G (Southern Biotech, Birmingham, AL, USA) and observed using a confocal laser scanning microscope (TCS SP5; Leica Microsystems GmbH, Wetzlar, Germany).

Western blot analysis. Cells was seeded into six-well plates at a density $1.0-2.0 \times 10^{6}$ cells/well and treated with alisertib $(0,0.1,1$ and $5 \mu \mathrm{M})$ for $24 \mathrm{~h}$. Cells were then lysed with ice-cold lysis buffer (1 mM phenylmethylsulfonylfluoride, $30 \mathrm{mM}$ Tris- $\mathrm{HCl} \mathrm{pH}$ 8.0, $150 \mathrm{mM} \mathrm{NaCl}$, protease/phosphatase

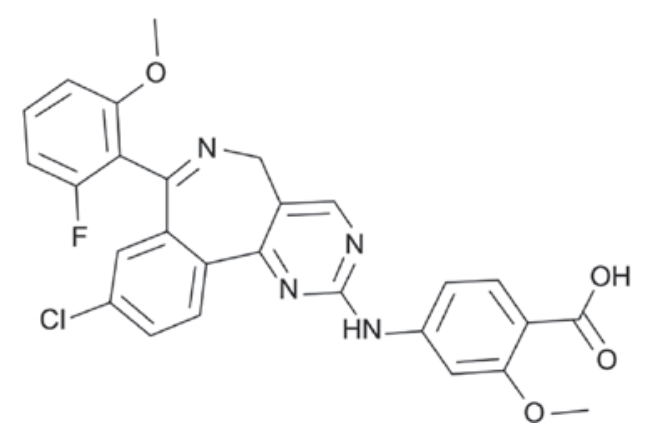

Figure 1. Chemical structure of alisertib.

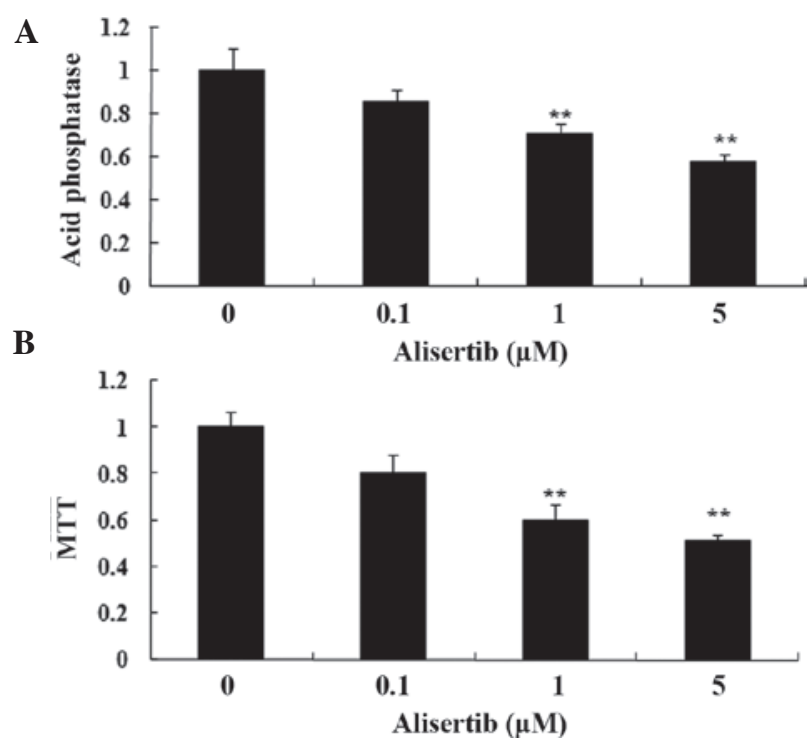

Figure 2. Alisertib suppresses the viability of REH cells. (A) Acid phosphatase and (B) MTT assays were used to assess the suppressive effects of alisertib on the viability of REH cells. Values are expressed as mean \pm standard error of the mean. ${ }^{* *} \mathrm{P}<0.01$ compared with $0 \mu \mathrm{M}$ alisertib-treatment group.

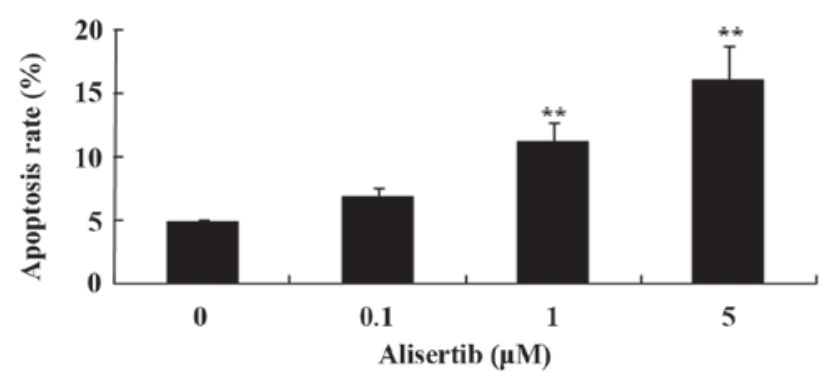

Figure 3. Alisertib induces autophagy in REH cells. The apoptotic rate was determined by Annexin V/propidium iodide double staining and flow-cytometric analysis. Values are expressed as mean \pm standard error of the mean ${ }^{* *} \mathrm{P}<0.01$ compared with $0 \mu \mathrm{M}$ alisertib-treatment group.

inhibitor cocktail and $1 \%$ nonidet P-40) for $30 \mathrm{~min}$. The lysate was then centrifuged at $12,000 \mathrm{x} \mathrm{g}$ for $10 \mathrm{~min}$ at $4^{\circ} \mathrm{C}$. Equal amounts of protein $(40-50 \mu \mathrm{g})$ were separated by sodium dodecyl sulfate-polyacrylamide gel (Sangon Biotech Co., Ltd., Shanghai, China) electrophoresis and transferred onto nitrocellulose membranes (Bio-Rad Laboratories, Inc., Hercules, CA, USA). Following incubation with mouse monoclonal anti-phosphorylated (p)-mTOR (cat. no. sc-293132; 


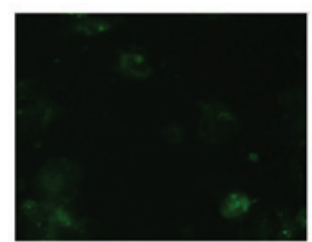

$0 \mu \mathrm{M}$

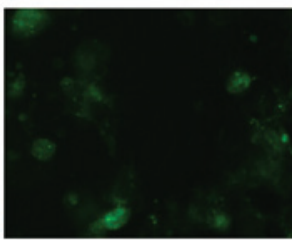

$0.1 \mu \mathrm{M}$



$1 \mu \mathrm{M}$

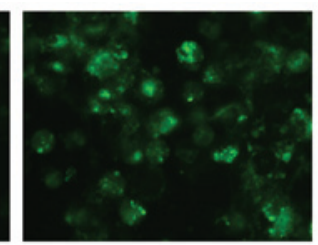

$5 \mu \mathrm{M}$

Alisertib

Figure 4. Alisertib induces autophagy of REH cells. Cells were immunostained with light chain 3B antibody (green). Magnification, x100.

1:1,000 dilution; Santa Cruz Biotechnology, Inc.), mouse monoclonal p-Akt (cat. no. sc-293125; 1:1,000 dilution; Santa Cruz Biotechnology, Inc.), rabbit polyclonal anti-p-adenosine monophosphate-activated kinase (AMPK) (cat. no. 4186; 1:2,000 dilution; Cell Signaling Technology, Inc.) and rabbit polyclonal anti- $\beta$-actin (cat. no. R1207-1; 1:800 dilution; HangZhou HuaAn Biotechnology Co., Ltd., Hangzhou, China). Subsequently, membranes were incubated with goat anti-rabbit (cat. no. A0208) and goat anti-mouse (cat. no. A0216) horseradish peroxidase-conjugated secondary antibodies from Beyotime Institute of Biotechnology (Haimen, China) at 1:5,000 dilution for $2 \mathrm{~h}$ at room temperature. Proteins were visualized using an enhanced chemiluminescence western blot detection system (EMD Millipore) and visualization was performed using Image J (version 1.31; National Institutes of Health, Bethesda, MA, USA).

Statistical analysis. Values are expressed as the mean \pm standard error. Statistical significance of the differences was analyzed by one-way analysis of variance followed by the StudentNewman-Keuls test. All statistical analysis was performed using SPSS 17.0 (SPSS, Inc., Chicago, IL, USA). All experiments were performed three times. $\mathrm{P}<0.05$ was considered to indicate a statistically significant difference.

\section{Results}

Alisertib reduces the viability of REH cells. The present study assessed the effects of alisertib on viability of REH cells using AP and MTT assays. Incubation of REH cells with alisertib at 1 and $5 \mu \mathrm{M}$ for $24 \mathrm{~h}$ significantly decreased the cell viability (Fig. 2). These results showed that alisertib potently decreases the viability of REH cells.

Alisertib induces apoptotic death of REH cells. Next, the present study explored the effects of alisertib on apoptosis of REH cells using flow cytometry following Annexin V-FITC and PI staining. As shown in Fig. 3, treatment with alisertib at $1 \mu \mathrm{M}$ for $24 \mathrm{~h}$ caused a two-fold increase of the apoptotic rate of REH cells, while $5 \mu \mathrm{M}$ alisertib increased the apoptotic rate by three-fold. These results demonstrated that alisertib exerts its anti-cancer effects on REH cells by inducing apoptosis.

Alisertib induces autophagy in REH cells. Since a previous study reported on the ability of alisertib to induce authophagy in osteosarcoma cells (7), the present study assessed whether alisertib induces autophagy in REH leukemia cells. As demonstrated by immunofluorescence microscopy, incubation of REH cells with alisertib at 1 and $5 \mu \mathrm{M}$ for $24 \mathrm{~h}$ resulted in marked expression of the autophagic marker LC3B (Fig. 4). These results indicated that alisertib may exert its anti-cancer effects via inducing autophagy of REH cells.

The mechanism of action of alisertib on leukemia cells may be mediated via deactivation of the MAPK/PI3K/Akt/mTOR pathway and activation of AMPK. To elucidate the possible molecular mechanisms via which alisertib induces apoptosis and autophagy in leukemic cells, its effects on the levels of p-mTOR, p-Akt, p-AMPK and p-p38 were assessed by western blot analysis. As shown in Fig. 5, treatment with 1 and $5 \mu \mathrm{M}$ alisertib significantly reduced the levels of $\mathrm{p}-\mathrm{mTOR}$ protein in REH cells; furthermore, the levels of p-Akt were reduced (Fig. 6), the levels of p-AMPK were increased (Fig. 7) and the levels of p-p38 (Fig. 8) were decreased ( $\mathrm{P}<0.01$ for all). These results suggested that deactivation of MAPK/PI3K/Akt/mTOR signaling and activation of AMPK may be involved in the mechanism of action of alisertib in leukemia cells.

\section{Discussion}

Studies have indicated a significant link between autophagy and neoplastic transformations of blood cells. Autophagy facilitates the removal of the mitochondria in red blood cells, which has an important regulatory role in early differentiation and maturation of red blood cells as well as in the development of myelodysplastic syndrome (8). In addition, autophagy may be the main cause of megakaryocyte dysfunction in myelodysplastic syndrome and idiopathic thrombocytopenic purpura (9). A variety of bioactive compounds and irradiation are known to induce autophagic death of leukemia cells. The results of the present study indicated that treatment with alisertib significantly reduced the viability of REH cells and promoted apoptotic cell death in a dose-dependent manner. Qi et al (10) indicated that alisertib inhibits the proliferation and induces apoptosis of T-Hodgkin lymphoma cells. Melichar et al (11) revealed that alisertib suppressed the grow of small-cell lung cancer, non-small-cell lung cancer, gastro-oesophageal adenocarcinoma, breast cancer and head and neck squamous-cell carcinoma. Furthermore, the results of the present study indicated that alisertib possesses activity against leukemia cells.

Autophagy is an important intracellular self-degradation mechanism, during which macromolecules and organelles within the cell are transported to the lysosomes for digestion and degradation by double-membrane vesicles, releasing free 
A

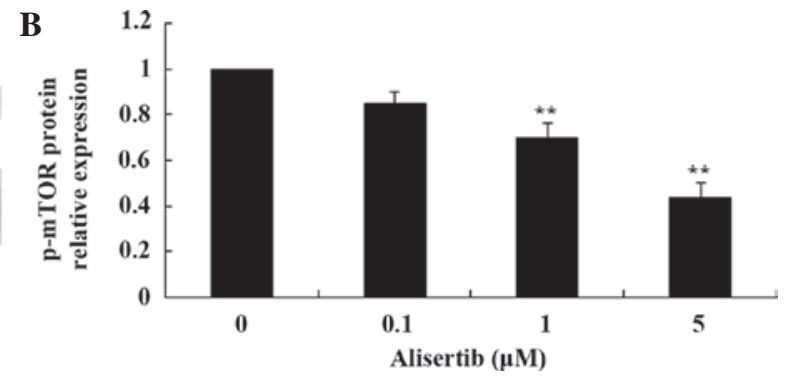

Figure 5. Alisertib suppresses autophagy through mTOR activation. (A) Representative western blot of p-mTOR protein levels in REH cells following Alisertib treatment and (B) quantified p-mTOR protein levels as determined by densitometric analysis normalized to $\beta$-actin. Values are expressed as mean \pm standard error of the mean. ${ }^{* *} \mathrm{P}<0.01$ compared with $0 \mu \mathrm{M}$ alisertib group. p-mTOR, phosphorylated mammalian target of rapamycin.

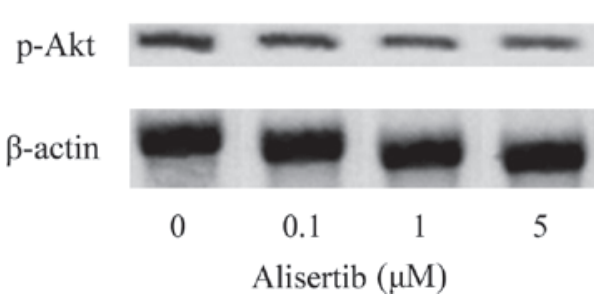

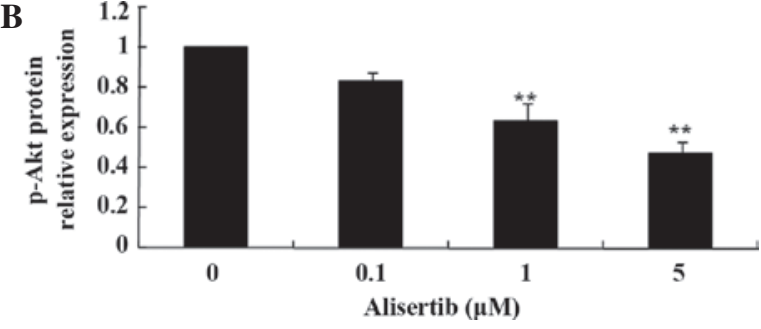

Figure 6. Alisertib suppresses autophagy through Akt activation. (A) Representative western blot of p-Akt protein levels in REH cells following Alisertib treatment and (B) quantified p-Akt protein levels as determined by densitometric analysis normalized to $\beta$-actin. Values are expressed as mean \pm standard error of the mean. ${ }^{* *} \mathrm{P}<0.01$ compared with $0 \mu \mathrm{M}$ alisertib group. $\mathrm{p}-\mathrm{Akt}$, phosphorylated Akt.
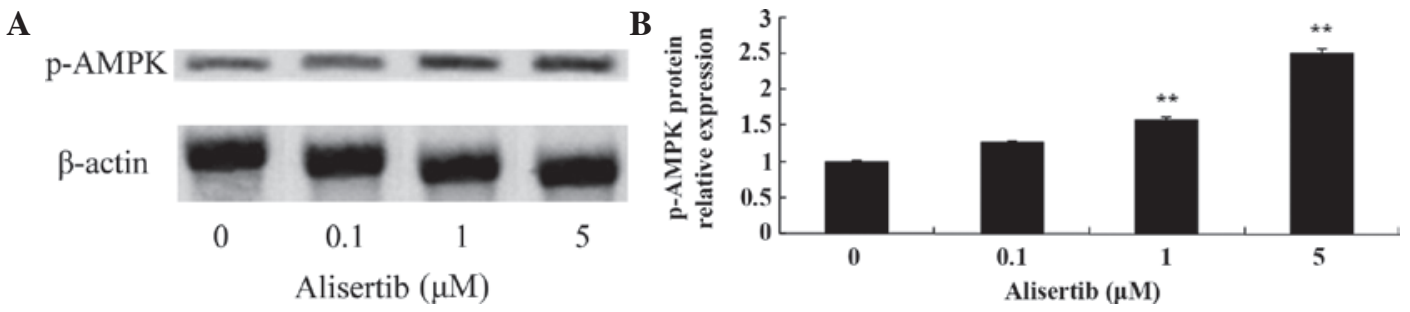

Figure 7. Alisertib induces autophagy through AMPK activation. (A) Representative western blot of p-AMPK protein levels in REH cells following Alisertib treatment and (B) quantified p-AMPK protein levels as determined by densitometric analysis normalized to $\beta$-actin. Values are expressed as mean \pm standard error of the mean. ${ }^{* *} \mathrm{P}<0.01$ compared with $0 \mu \mathrm{M}$ alisertib-treatment group. p-AMPK, phosphorylated 5'-adenosine monophosphate-dependent kinase.

A

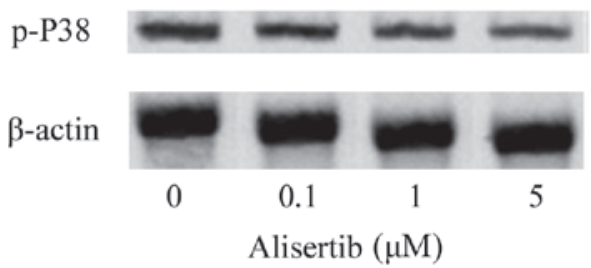

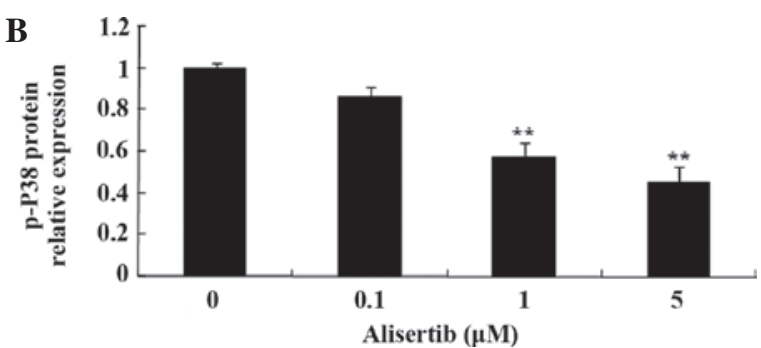

Figure 8. Alisertib induces autophagy through p38 activation. (A) Representative western blot of p-P38 protein levels in REH cells following Alisertib treatment and (B) quantified p-P38 protein levels as determined by densitometric analysis normalized to $\beta$-actin. Values are expressed as mean \pm standard error of the mean. ${ }^{* *} \mathrm{P}<0.01$ compared with $0 \mu \mathrm{M}$ alisertib-treatment group. p-P38, phosphorylated $\mathrm{P} 38$.

small molecules for recycling in the cell (12). Almost all types of eukaryotic cell have the capacity to undergo autophagy (13). It is known that this mechanism has an important role in cell physiological processes, including cell development, differentiation, senescence and death, while the specific mechanisms have remained to be fully elucidated. Autophagy can be divided into macro-autophagy, micro-autophagy and chaperone-mediated autophagy (14). In contrast to the ubiquitin protein degradation system, autophagy can avert pathogenic attacks as well as cell damage caused by physical and chemical factors, apart from acting as a energy retrieval by self digestion (15). Autophagy also acts in a power switch-like manner to change the threshold level of the regulation of cell survival in response to apoptotic stimuli. In the embryonic development period as 
well as in adult organisms, excessive levels of autophagy lead to autophagic death (16). The findings of the present study showed that alisertib dose-dependently induced autophagy in REH cells. Wang et al (17) reported that alisertib induces cell cycle arrest and autophagy of human pancreatic cancer cells through PI3K/Akt/mTOR. Furthermore, Ding et al (18) also reported that alisertib induced apoptosis and autophagy of human epithelial ovarian cancer cells. These results suggested that induction of autophagy represents a major mechanism of action of alisertib, which is a desired mechanism of cancer treatments.

mTOR is an important factor regulating cell growth and proliferation, and influences processes including nutrition-associated molecules in signal transduction, regulation of protein translation and cell cycle progression; furthermore, activated mTOR is an inhibitor of autophagy (19). The initiation of autophagy is mainly inhibited by molecules targeting mTOR, including TOR complex 1 (TORC1) and type I PI3K (20). Signaling of growth factors within the cell and information regarding the nutrient status and energy levels are conveyed to TORC1 through Type I PI3K and Akt/protein kinase B pathways, resulting in the activation of TORC1 at an appropriate level, thereby activating mTOR to inhibit autophagy (21). Therefore, the activity of mTOR is influenced by the activity of TORC1 activity to regulate the level of autophagy (22). The results of the present study revealed that alisertib decreased the phosphorylation/activation of mTOR as well as that of Akt and p38, thereby inducing autophagy in REH cells, which was in line with the findings of a previous study (17). Furthermore, alisertib significantly activated AMPK in REH cells. Yuan et al (23) indicated that alisertib induces apoptosis and autophagy via activation of AMPK and suppression of p38 MAPK in human gastric cancer. These results indicated that alisertib induces autophagy in REH cells and other cancer cell types via activation of AMPK and suppression of the PI3K/Akt/mTOR pathway.

In conclusion, the present study reported that alisertib exerted marked anti-cancer effects against the REH leukemia cell line by suppressing cell grow, promoting apoptosis and inducing autophagy. The underlying molecular mechanism of the autophagy-inducing effects of alisertib in REH cells were indicated to include the deactivation of the PI3K/Akt $/ \mathrm{mTOR}$ pathway and activation of AMPK.

\section{References}

1. Yi B, Zhang M, Schwartz-Albiez R and Cao Y: Mechanisms of the apoptosis induced by CD176 antibody in human leukemic cells. Int J Oncol 38: 1565-1573, 2011.

2. Manzotti G, Mariani SA, Corradini F, Bussolari R, Cesi V, Vergalli J, Ferrari-Amorotti G, Fragliasso V, Soliera AR, Cattelani S, et al: Expression of p89 (c-Mybex9b), an alternatively spliced form of c-Myb, is required for proliferation and surviva of p210BCR/ABL-expressing cells. Blood Cancer J 2: e71, 2012.

3. Thapalia BA, Zhou Z and Lin X: Autophagy, a process within reperfusion injury: An update. Int J Clin Exp Pathol 7: 8322-8341, 2014.

4. Huang Z, Ye B, Dai Z, Wu X, Lu Z, Shan P and Huang W: Curcumin inhibits autophagy and apoptosis in hypoxia/reoxygenation-induced myocytes. Mol Med Rep 11: 4678-4684, 2015.

5. Su X, Wang X, Liu Q, Wang P, Xu C and Leung AW: The role of Beclin 1 in SDT-induced apoptosis and autophagy in human leukemia cells. Int J Radiat Biol 91: 472-479, 2015.

6. Manfredi MG, Ecsedy JA, Chakravarty A, Silverman L, Zhang M, Hoar KM, Stroud SG, Chen W, Shinde V, Huck JJ, et al: Characterization of Alisertib (MLN8237), an investigational small-molecule inhibitor of aurora A kinase using novel in vivo pharmacodynamic assays. Clin Cancer Res 17: 7614-7624, 2011.
7. Niu NK, Wang ZL, Pan ST, Ding HQ, Au GH, He ZX, Zhou ZW, Xiao G, Yang YX,Zhang X, et al: Pro-apoptotic and pro-autophagic effects of the Aurora kinase A inhibitor alisertib (MLN8237) on human osteosarcoma U-2 OS and MG-63 cells through the activation of mitochondria-mediated pathway and inhibition of p38 MAPK/PI3 K/Akt/mTOR signaling pathway. Drug Des Devel Ther 9: 1555-1584, 2015.

8. Bunaciu RP, Tang T and Mao CD: Differential expression of Wnt13 isoforms during leukemic cell differentiation. Oncol Rep 20: 195-201, 2008.

9. Wang Y, Lin Z, Huang H, He H, Yang L, Chen T, Yang T, Ren N, Jiang $\mathrm{Y}, \mathrm{Xu} \mathrm{W}$, et al: AMPK is required for PM2.5-induced autophagy in human lung epithelial A549 cells. Int J Clin Exp Med 8: 58-72, 2015.

10. Qi W, Spier C, Liu X, Agarwal A, Cooke LS, Persky DO, Chen D, Miller TP and Mahadevan D: Alisertib (MLN8237) an investigational agent suppresses Aurora A and B activity, inhibits proliferation, promotes endo-reduplication and induces apoptosis in T-NHL cell lines supporting its importance in PTCL treatment. Leuk Res 37: 434-439, 2013.

11. Melichar B, Adenis A, Lockhart AC, Bennouna J, Dees C, Kayaleh O, Obermannova R, DeMichele A, Zatloukal P, Zhang B, et al: Safety and activity of alisertib, an investigational aurora kinase A inhibitor, in patients with breast cancer, small-cell lung cancer, non-small-cell lung cancer, head and neck squamous-cell carcinoma, and gastro-oesophageal adenocarcinoma: A five-arm phase 2 study. Lancet Oncol 16: 395-405, 2015.

12. Zhang J, Wu K, Xiao X, Liao J, Hu Q, Chen H, Liu J and An X: Autophagy as a regulatory component of erythropoiesis. Int J Mol Sci 16: 4083-4094, 2015.

13. Moon HS, Kim B, Gwak H, Suh DH and Song YS: Autophagy and protein kinase RNA-like endoplasmic reticulum kinase (PERK)/eukaryotic initiation factor 2 alpha kinase (eIF2 $\alpha$ ) pathway protect ovarian cancer cells from metformin-induced apoptosis. Mol Carcinog Feb 7, 2015 (Epub ahead of print).

14. Condello M, Caraglia M, Castellano M, Arancia G and Meschini S: Structural and functional alterations of cellular components as revealed by electron microscopy. Microsc Res Tech 76: 1057-1069, 2013.

15. Krampe $S$ and Boles E: Starvation-induced degradation of yeast hexose transporter Hxt7p is dependent on endocytosis, autophagy and the terminal sequences of the permease. FEBS Lett 513: 193-196, 2002.

16. Lamark T and Johansen T: Autophagy: Links with the proteasome. Curr Opin Cell Biol 22: 192-198, 2010.

17. Wang F, Li H, Yan XG, Zhou ZW, Yi ZG, He ZX, Pan ST, Yang YX, Wang ZZ, Zhang X, et al: Alisertib induces cell cycle arrest and autophagy and suppresses epithelial-to-mesenchymal transition involving PI3K/Akt/mTOR and sirtuin 1-mediated signaling pathways in human pancreatic cancer cells. Drug Des Devel Ther 9: 575-601, 2015.

18. Ding YH, Zhou ZW, Ha CF, Zhang XY, Pan ST, He ZX, Edelman JL, Wang D, Yang YX, Zhang X, et al: Alisertib, an Aurora kinase A inhibitor, induces apoptosis and autophagy but inhibits epithelial to mesenchymal transition in human epithelial ovarian cancer cells. Drug Des Devel Ther 9: 425-464, 2015.

19. Jung CH, Kim H, Ahn J, Jung SK, Um MY, Son KH, Kim TW and Ha TY: Anthricin Isolated from Anthriscus sylvestris (L.) Hoffm. Inhibits the growth of breast cancer cells by inhibiting Akt/mTOR signaling and its apoptotic effects are enhanced by autophagy inhibition. Evid Based Complement Alternat Med 2013: 385219, 2013.

20. Badura S, Tesanovic T, Pfeifer H, Wystub S, Nijmeijer BA, Liebermann M, Falkenburg JH, Ruthardt M and Ottmann OG: Differential effects of selective inhibitors targeting the $\mathrm{PI} 3 \mathrm{~K} / \mathrm{AKT} / \mathrm{mTOR}$ pathway in acute lymphoblastic leukemia. PLoS One 8: e80070, 2013.

21. Qin L, Wang Z, Tao L and Wang Y: ER stress negatively regulates $\mathrm{AKT} / \mathrm{TSC} / \mathrm{mTOR}$ pathway to enhance autophagy. Autophagy 6: 239-247, 2010.

22. Vakana E, Sassano A and Platanias LC: Induction of autophagy by dual mTORC1-mTORC2 inhibition in BCR-ABL-expressing leukemic cells. Autophagy 6: 966-967, 2010.

23. Yuan CX, Zhou ZW, Yang YX, He ZX, Zhang X, Wang D, Yang T, Wang NJ, Zhao RJ and Zhou SF: Inhibition of mitotic Aurora kinase A by alisertib induces apoptosis and autophagy of human gastric cancer AGS and NCI-N78 cells. Drug Des Devel Ther 9: 487-508, 2015. 